\title{
Verstopfung des Magens von Geisteskranken durch Fremdkörper.
}

\author{
Von \\ Dr. v. Schleiß-Löwenfeld. \\ Mit 3 Textabbildungen. \\ (Eingegangen am 21. September 191\%.)
}

Fremdkörper im Magen Geisteskranker sind im allgemeinen keine seltene Erscheinung. In den Jahrbüchern der chirurgischen Kliniken finden sich Berichte von Fremdkörpern aller denkbaren Arten, welche aus Magen oder Darm operativ entfernt wurden. Um nur einige Beispiele anzuführen: in der Deutschen med. Wochenschr. (1897) gibt der Verfasser (Dr. Fricken) 53 Fälle an (veröffentlicht von Credé, Langenbecksch. Archiv 33); unter denselben finden sich einige Fälle, bei denen größere Mengen von Fremdkörpern in Betracht kommen: Ein 20 jähr. Mädchen mit einem Haarknäuel von $120 \mathrm{~g}$ Gewicht; ein männlicher, chronisch-manischer Kranker, welcher 192 Nägel und Knöpfe und Haarknäuel, zusammen 1 Pfund $91 / 2$ Unzen im Magen hatte; die Zeit des Verweilens derselben war nicht zu bestimmen. Der Fall Frik kens betraf eine 32 jährige Frau mit hysterischen Beschwerden, welche 37 Stücke aller Art und Größe in selbstmörderischer Absicht verschluckt hatte, ein Gewicht von $261,85 \mathrm{~g}$, und welche sich ca. 3 Monate im Magen befanden. Der Beginn des Verschluckens infolge Melancholie mit Selbstmordabsicht lag in diesem Falle schon ca. $1^{1 / 2}$ Jahre vor der Operation; von kleineren Gegenständen, Knöpfen, Nadeln usw. - von denen wohl viele per vias naturales abgegangen sind - kam sie allmählich zu größeren. Die Beschwerden der Frau waren anfangs gering; sie dauerten nach Heilung der geistigen Erkrankung fort und steigerten sich zu heftigen Schmerzen, Kräfteverfall, Erbrechen, Unfähigkeit der Nahrungsaufnahme, Magenabsceß infolge Perforation einer Häkelnadel. Der Nachweis der Fremdkörper gelang erst während der Operation. Ein weiterer Fall von jahrelangem Verschlingen von Fremdkörpern wird in der Deutschen med. Wochenschr. von Dr. B. Beck mitgeteilt. Er betrifft einen 32 jähr. Tagelöhner (Alkoholisten); derselbe verschluckte seit ca. 5 Jahren Nägel, Eisenstifte und dgl; allmählich kam er zu mehr und zu größeren Gegenständen, besonders Taschenmessern (infolge sog. „Bierwetten"). Alle Gegenstände verursachten lebhafte Schmerzen 
im Epigastrium, schleimig-blutiges Erbrechen; die meisten Dinge gingen per vias naturales ab; nur von den Taschenmessern mußten mehrere durch Operation entfernt werden. Verfasser sagt, daß, selten Gastrotomie zur Entfernung der Fremdkörper notwendig wurde . . ., nicht als ob das Eindringen von Fremdkörpern in den Magen selten wäre - im Gegenteil, zahlreiche Fälle sind in der Literatur angegeben, aber selten ist Operation notwendig, weil die Gegenstände ... per vias naturales den Magen verlassen ... ohne Störung"“.

Die im vorstehenden angegebenen Fälle wurden, cbwohl sie keine ,Verstopfung“ des Magens mit Fremdkörpern betreffen, herausgegriffen, weil sie für viele andere dieser Art typisch sind und weil sie einige Vergleichungspunkte mit unseren Fällen darbieten. Diese seien nun in der Reihenfolge, wie sie zur Beobachtung kamen, in möglichster Abkürzung der Krankengeschichten wiedergegeben:

I. Ba., U., weibliche Kranke, wurde im April 1904 im Alter von 26 Jahren im Allgemeinen Krankenhause 1. I. in München aufgenommen. Es handelt sich um einen Fall progressiver Katatonie. Anfangs -. d. h. die ersten Monate nach ihrer Aufnahme - zeigte die Kranke noch eine zeitweilige gedrückte Gemütsstimmung, ängstliche Erregtheit und Unruhe; sie gab wenigstens einige Male Auskunft auf Befragen, wenngleich bereits der Negativismus, blind-wütendes Abwehren und verwirrtes Schreien schon vorherrschend waren. Ihre Äußerungen betrafen fast ausschließlich religiöse Dinge; ihren verwirrten, mit Gebetbruchstücken, Bibel- und Katechismusstellen untermischten Reden war mit Mühe zu entnehmen, daß sie sich für eine große Sünderin, eine Diebin, für ewigverdammt halte. Sie drängte gewaltsam nach Türen und Fenstern, zerriß Kleider und Wäsche, lief nackt umher, nahm mangelhaft Nahrung, war Tag und Nacht laut und lärmend, durch Narkotica wenig zu beeinflussen.

Am 5. X. 1904 nach Gabersee transferiert, verfiel sie in einen wochenlangen stuporösen Zuṣtand, aus dem sie mit gesteigerter Erregtheit erwachte. Die Demenz machte schnelle Fortschritte. Allmählich verschwand die ängstliche Stimmung und machte einer ziellosen, explosiv ausbrechenden Erregtheit Platz; Pat. gehörte bald zu den störendsten, dabei höchst unreinlichen und zerstörungssüchtigen Kranken. Sie mußte stets im Bett gehalten werden, da sie.., ,sich höchst erregt gebärdete, halb religiöse, halb sexuelle, absurde Wahnideen in einem eigentümlichen, singenden Tone und manieriert-hochdeutscher, pathetischer Sprechweise äußerte, unter heftigen Gestikulationen und Grimassen ein schwärmerisches, exaltiertes Wesen zur Schau tragend." Ihre stereotypen Redensarten wiederholte sie unzählige Male, z. B. „Lassen Sie mich zu meinem Jesus! Zu meinem Jesus!..." usw. „Unrecht Gut gedeiht niemals! gedeiht niemals!..." usw. „Ich allein bin der Herr! Ich allein bin der Herr!" usw. „Nein, ihr sollt nicht töten, spricht der Herr ..." „Lasset ihn kommen, den Herrn!" usw. Jede, noch so kurze Anrede an die Pat. löste einen förmlichen Anfall von Schreien (ähnlicher Redensarten, wie angegeben) aus, wobei Pat. auf die Knie stürzte, an Ärzte und Pflegerinnen sich anklammerte und dann $1 / 2-1$ Stunde lang weiter schrie in der bezeichneten Weise. Dieser Zustand hielt im wesentlichen an bis zu ihrem Tode, nur wurde das Schreien später weniger laut infolge der zunehmenden körperlichen Schwäche. Die Kranke wurde immer unreinlicher und zerstörungssüchtiger, sie widerstrebte jeder Untersuchung und Behandlung und überhaupt allen Anforderungen, die an sie gestellt wurden. Sie mußte häufig isoliert werden, da sie 
durch ihr sinnloses Schreien und - mehr noch - durch ihre hastigen Bewegungen, wilden Gestikulationen und Grimassen die Mitpatientinnen erregte. Körperlich magerte die Pat. mehr und mehr ab, trotz (oder wegen ?) des krankhaften Heißhungers, mit dem sie alles EBbare verschlang und den Mitpatientinnen wegzunehmen suchte. Von August 1906 an traten häufige, doch nur vorübergehende Diarrhöen auf, „,meist nur am Morgen und während des Tages ohne weitere Behandlung wieder zurückgehend". Seit Ende des Jahres 1907 machte sie den Eindruck einer phthisischen Kranken: „Hochgradige Abmagerung, anämisches, eingefallenes Äußeres, Husten ohne Expektorationen; unter der rechten Unterkinnlade eine verhärtete, haselnuBgroße Lymphdrüsenschwellung." (Genauere Untersuchung bei dem Widerstreben der Pat. nicht durchführbar.) Die Nahrung bestand seit November 1906 in Flüssigkeiten (Milch, Suppe usw.), welche sie in reichlicher Menge bis 3 Tage vor ihrem Tode zu sich nahm. Sie lag die letzten 3 Tage unbeweglich, aufs äußerste abgemagert, leichenähnlich blaß da, doch fehlte die bei Phthisikern gewöhnliche Schweratmigkeit. Unter allmählichem Aufhören von Puls und Atmung trat der Tod der Pat. ein am 5. II. 1908.

Die Sektion ergab außer nebensächlichen Befunden an Lungen, Herz, Nieren und Gehirnhäuten einen überraschenden Befund am Magen. Er lag bei Eröffnung

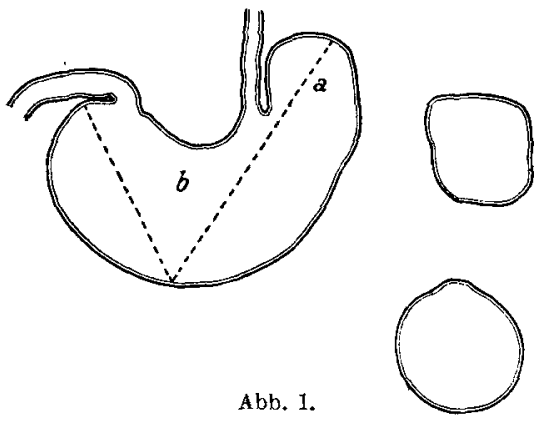
der Bauchhöhle zu etwa $2 / 3$ seines stark vergrößerten Volumens vor, in weißgrauer Farbe, den Anblick eines prall ausgestopften Kissens (einer sog. ,,Schlum. merrolle") darbietend. Der obere Teil des Fundus war auffällig nach oben ausgebuchtet und unter das Zwerchfell hinaufged rängt, einen fast viereckigen Querschnitt zeigend. Die große Kurvatur reichte fast bis 3 Querfinger unter den Nabel; die kleine Kurvatur war stark gekrümmt, so daß Eingang und Ausgang des Magens dadurch einander genähert schienen. Der Mageninhalt quoll beim Ein. schneiden sofort hervor; er hatte ein Gewicht von $1700 \mathrm{~g}$ und bestand zum größten Teil aus Holzwollfasern, dazu Frauenhaaren, verfilzt zu einem festen, harten, nur mit Gewalt zerreißbaren Knäuel. Die stärkste Verfilzung war an der tiefsten Stelle der großen Kurvatur. Es fanden sich zwischen den Holzfasern auch zusammengeballte Stückchen von Wachsleinwand (Betteinlage bei Unreinlichen!) von $10-15 \mathrm{~cm}$ Länge, Streifchen von Bettdecken, Läppchen von Wäsche, Watte, Matratzenstrickchen, auch Eicheln, von welchen die Schale gut erhalten, der Kern aber verschwunden (verdaut) war. Der kleinen Kurvatur entlang fand sich noch etwas flüssiger Speisebrei und zeigte den Weg, welchen die - flüssige Nahrung in der letzten Zeit genommen hatte; der tieferliegende Inhalt des Magens war stark durchtränkt von Flüssigkeit. Die Schleimhaut des Magens war ganz blaß und blutleer, ohne Falten, dünn und atrophisch, doch ohne jeden geschwürigen Defekt; im unteren, dem Pylorus benachbarten Teil fanden sich 8- 10 linsengroße, beetartige, in Gruppen stehende Erhabenheiten, die auf dem Durchschnitt eine gleichmäßige, rötliche Färbung zeigten. Die Magenwand war nur im Pylorusteil etwas verdickt, sonst überall gleichmäßig dünn und wie über den Inhalt gespannt. Die Oesophagusschleimhaut war in einer Höhe von $5 \mathrm{~cm}$ über der Kardia injiziert und auffallend verdickt und rauh. Dje Länge des gefüllten Magens betrug (mit unbiegsamem Maßstab gemessen) $50 \mathrm{~cm}$ (sjehe obenstehende schematische Zeichnung, Abb. 1). 
Vor allem drängt sich die Frage auf: wie konnte sich eine solche Menge von Fremdkörpern im Magen ansammeln, daß der Magen dadurch buchstäblich ausgestopft erschien? Zweifellos war die Ausfüllung eine allmähliche, langsame, einen sicheren Anhaltspunkt hierfür geben die Eicheln, welche in den verfilzten Teilen eingelagert waren: Patientin konnte dieselben nur in einem Garten gefunden haben, den sie seit September 1907 nicht mehr betreten hatte; daraus läßt sich schlieBen, daß mindestens 4-41/2 Monate vor dem Tode der Patientin die Fremdkörperansammlung begonnen hat; wahrscheinlich war die Dauer eine weit größere, vielleicht über ein Jahr (nach den Verdauungsstörungen und der Abmagerung zu schließen, seit August 1906). Die aufgenommenen Flüssigkeiten durchtränkten den Mageninhalt, die Holzfasern quollen dadurch auf; das Utberschüssige floß entlang der kleinen Kurvatur ab, was durch die tiefere Lage des Pylorus zur Cardia erleichtert wurde. In der Pylorusgegend fanden sich auch die Erscheinungen einer chronischen Reizung der Schleimhaut, der erwähnte „Etat mamelonné". Nirgends aber ließen sich Nekrosen, Druckbrandsymptome konstatieren, was doch bei dem Gewicht des Fremdkörpertumors $1700 \mathrm{~g}$ - erklärlich gewesen wäre. Im Darm ließ sich nirgends eine Spur von Fremdkörpern nachweisen.

Der zweite Fall, Mon., B., wurde, 22 Jahrè alt, im März 1906 in die Psychiatrische Klinik München, von da nach Eglfing und von da am 21. IX. 12 nach Gabersee verbracht. Ohne nachweisbare Ursache erkrankte Pat. plötzlich, fing an, alles verkehrt zu machen, Gegenstände zu zertrümmern, verwirrt zu reden. In die Klinik gebracht, war sie grob und wiederstrebend: ,Sie wehrte die Untersuchung $a b$, antwortete auf Befragen nicht, schimpfte dagegen in den gemeinsten Ausdrücken. Allem setzte sie den hartnäckigsten Widerstand entgegen; passiven Bewegungen widerstrebte sie nach Möglichkeit. Ins Bad gebracht, schimpfte sie erst, dann sa $B$ sie ruhig, kümmerte sich nicht um die Umgebung, ließ in nahezu regelmäßigen Abständen ein minutenlanges, unmotiviertes Lachen erschallen. “ . ..,,Tagelang verhielt sie sich ganz abweisend, mutazistisch, schloß bei Annäherung des Arztes die Augen, versteckte sich in den Kissen, reagierte weder auf Nadelstiche noch auf Schokolade, schlug bei längerer Bemühung nach dem Referenten.“ Allmählich wurde sie zeitweise etwas zugänglicher: „Pat. ist wenig zugänglich; wenn es aber gelingt, sie zu fixieren, so wird sie sehr geschwätzig, macht allerhand Witze, Wortreimereien; sie neckt gern andere Kranke." Bezüglich Sinnestäuschungen war nur einmal eine Äußerung aus der Kranken herauszubringen: es seien viele Gespenster um sie herum. Die Kranke blieb andauernd ,äußerst unsauber, gereizt, aggressiv, dabei zu Scherzen geneigt; eine Unterhaltung nie möglich".

Die Geisteskrankheit der Pat. wurde anfangs als manisch-depressives Irresein diagnostiziert; allmählich aber trat ihre Zugehörigkeit zur Dementia praecox klar hervor, nur die Frage, ob es sich um Hebephrenie oder Katatonie handle, konnte Zweifel hervorrufen. Doch scheint die Katatonie als das Wahrscheinlichere; der andauernde Negativismus, die explosiven Erregungen sprechen dafür, abgesehen von den schon bemerkten Stereotypien, dem unbezähmbar-heftigen, wilden, dabei ziellosen Gebaren. Bei ihrer Transferierung nach Gabersee (September 1912) war die Demenz bereits weit vorgeschritten; echte Affekte, moralische und 
ethische Gefühle erschienen gänzlich geschwunden. „Pat. ist völlig unzugänglich; meist liegt sie unter der Decke verborgen, nimmt an nichts Anteil; in ihren Äußerungen zeigt sie keine Ideenflucht, sondern stete Wiederholung der nämlichen, zum Teil kindischen Redewendungen, zum Teil wüsten Schimpfereien"; „für Ärzte und Personal immer abweisend; sie streckt die Zunge heraus, schreit und lacht ausgelassen; wenn sie von ihrem ziellosen Umherrennen im Saale und boshaften Neckereien von Mitpatienten abgehalten wird, rauft sie wütend; sie muß häufig, besonders nachts isoliert werden. Die Nahrungsaufnahme war immer reichlich, hastig und gierig". Ihr körperlicher Zustand wurde bei der Aufnahme als ,,mittelgroß, kräftig, gut genährt, ohne Entwicklungshemmungen und Störung der inneren Organe" (soweit Untersuchung möglich) bezeichnet. Schon im November 1912 wurde ihr ,gelblich-fahles, anämisches Aussehen" beobachtet, ungefähr zu gleicher Zeit ,die Gewohnheit, Spitzen ihrer Haare abzubeißen und zu verschlucken, auch Wolle aus den Decken, Holzwollfasern und sonstiges Zeug". Im März 1913 stellten sich Diarrhöen und Erbrechen ein; ihr Appetit resp. Gier und Hastigkeit bei der Nahrungsaufnahme war dadurch nicht beeinträchtigt. Allmählich nahm das Erbrechen zu; ihr Aussehen wurde immer schlechter, fahl, lehmgelb (ohne daß Symptome organischer Erkrankung, z. B. seitens der Lungen, nachweisbar gewesen wären). Das Erbrechen (17. IV. 13) ,... stellte sich fast jeden Tag unmittelbar nach dem Essen ein, ein- bis zweimal; dabei wurde das Gegessene ohne vorhergehendes Würgen, gußweise wieder erbrochen, ohne Spur von Verdauungserscheinung". Pat. erhielt flüssige Kost, welche sie mit gleicher Gier zu sich nahm; trotzdem verschlimmerte sich das Erbrechen immer mehr: es trat täglich drei- bis viermal auf, immer ganz kurze Zeit $(1 / 4-1 / 2$ stunde) nach dem Essen. Trotz heftigen Widerstrebens der Pat. ließ sich (Anfang April 1913) ein Tumor in der Magengegend nachweisen: ,steinhart, kindskopfgroß, an der linken Bauchseite, bis 3 Querfinger unter den Nabel herab reichend, verschieblich, mit den Bauchdecken nicht verwachsen; Schmerzempfindung äußerte Pat. bei der Untersuchung nicht" (bei dem Widerstreben der Pat. nicht sicher zu entscheiden). Zu dem fast kachektisch zu nennenden Aussehen der Pat. kam eine rapide Zunahme der Schwäche und Abmagerung. Seit April 1913 lag sie fast immer zusammengekauert im Bett, wurde aber bei Annäherung eines Arztes oder einer Pflegerin sofort abweisend und gewalttätig, soweit ihre Kräfte es zuließen. Im Stuhl wurde nichts Besonderes bemerkt bis 3 Tage vor ihrem Tode: dann traten Diarrhöen mit deutlicher Blutbeimengung auf. Ohne sonstige auffallende Erscheinungen trat am 13. V. 1913 der Tod der Pat. ein.

Die Sektion ergab hochgradige Anämie sämtlicher Brust- und Bauchorgane. Die Knochen des Rumpfes, namentlich Sternum, Rippen, Wirbelsäule waren sehr weich, ließen sich mit dem Messer schneiden, das Knochenmark schmierig, ähnlich der Milzpulpa. Kurz: die Symptome der Osteomalacie waren deutlich nachweisbar. Der Magen ,,lag bei der Eröffnung der Bauchhöhle vor, aufgetrieben und ausgedehnt, bis unter den Nabel reichend, hart anzufühlen, schwer. Er enthielt ein verfilztes Konglomerat von Fremdkörpern und ca. $3 / 4$ Liter weißgrauer Flüssigkeit, welche sich durch den Oesophagus entleeren ließ. Der feste Inhalt wog $2250 \mathrm{~g}$. Die Maße des (gefüllten) Magens waren: Länge $40 \mathrm{~cm}$, Breite $30 \mathrm{~cm}$, Dicke $10 \mathrm{~cm}$. Die Fremdkörpermasse - aus Holzfasern, Wolle- und Leinwandfetzen und Ähnlichem bestehend - lag der hinteren und unteren Magenwand an, während (im Leben, bei der sitzenden und liegenden Stellung der Pat.) die genannte Flüssigkeit die kleine Kurvatur und die vordere Magenwand bespülte. Der Pylorus war durch Fremdkörper ganz verstopft; der angrenzende Darmabschnitt noch bis auf $40 \mathrm{~cm}$ Länge mit denselben Fremdkörpern ausgefüllt und erweitert noch weitere $30 \mathrm{~cm}$ darüber hinaus war der Darm noch stark erweitert, so als wären auch hier Fremdkörper gewesen, welche sich vielleicht Josgetrennt und 
durch den Stuhl entleert hatten. Die Form des Magens war im allgemeinen der normalen gleich geblieben, ebenso seine Lage zu den übrigen Organen. Die Schleimhaut - außer daß sie sehr dünn und blutleer war - zeigte keine besonderen Veränderungen. Die Dünndarmschleimhaut war injiziert und mit wässerigem, blutigem Schleim belegt; der Dickdarm ohne besondere Veränderung, enthielt nur wenig flüssigen Kot (Abb. 2).

In diesem (II.) Falle konnte die Diagnose auf Fremdkörperansammlung im Magen schon zu Lebzeiten der Patientin gestellt werden - mit Wahrscheinlichkeit; denn natïrlich war die Möglichkeit eines Carcinoms nicht

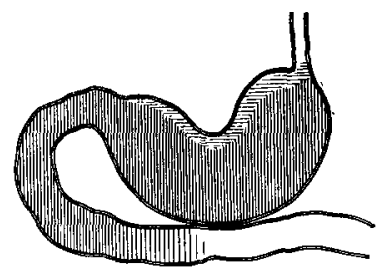

Abb. 2. von der Hand zu weisen. Einigen Anhaltspunkt gewährte die Art des Erbrechens. Wie schon angegeben, erfolgte dasselbe gußweise, kurz nach der Nahrungsaufnahme, auch wenn dieselbe nur in Wasser bestand, ohne die sonstigen Begleiterscheinungen des Erbrechens: ohne Würgen, ohne krampfhafte Gesichtsverziehungen. Man konnte an das Utberllaufen eines überfüllten Gefäßes denken. Das Erbrechen war ohne Blut-, Schleim- und sonstige Beimengungen.

Für die Dauer der Anschoppung ließ sich in diesem Falle kein bestimmter Zeitpunkt feststellen; das erdig-fahle Aussehen und die Verdauungsstörungen wurden zuerst im November 1912 angegeben. Der allgemeine Eindruck, den die Form und der Inhalt des Magens hervorrief und der Vergleich mit dem Falle I, war der, daß hier die Anschoppung vielleicht seit nicht ganz so langer Zeit wie im Fall I, doch auch schon seit mindestens 4-5 Monaten stattgefunden hatte. Die Ausfuillung der dem Pylorus angrenzenden Darmabschnitte zeigt, daß hier seit längerem ein vollständiger Abschluß des Magens für feste Nahrungsstoffe bestand, während Flüssigkeiten durch das Holzfasergemenge noch durchsickerten.

Nit Sicherheit war bei diesem Falle zu konstatieren, daß der krankhafte Heißhunger der Patientin durch die Anschoppung des Magens nicht gemildert wurde; selbst als der Tumor bereits nachgewiesen war und längst nur mehr flüssige Nahrung aufgenommen werden konnte, dauerte der Heißhunger noch an.

Bei diesem Falle (II) waren auch die Symptome von Osteomalacie deutlich nachweisbar: Sternum-, Rippen- und Wirbelknochen waren leicht mit dem Messer schneidbar, nicht dagegen der Schädelknochen, wie dies bei zahlreichen, in Gabersee beobachteten, vorgeschrittenen Fällen die Regel war; der vorliegende Fall befand sich offenbar im Anfangsstadium der Osteomalacie.

Im folgenden, III. Fall wurde eine vollständige Verstopfung des Magens nicht erreicht, insofern er wenigstens für Flüssigkeiten durchgängig blieb; die Kranke starb an einer hinzugekommenen Tuber- 
kulose der Lungen. Doch war auch hier die Ausfüllung des Magens mit Fremdkörpern eine nahezu vollständige.

III. Hu., J. wurde im Alter von 29 Jahren in Gabersee aufgenommen im Oktober 1912. Die ersten Erscheinungen geistiger Störung traten im September 1911 (also im 28. Lebensjahre der Pat.) auf; Pat. befand sich damals im Krankenhause zu P. wegen „hysterischen Tremors beider Arme und anderer Anzeichen hysterischen Charakters"; nach ca. 6 Wochen „,nur wenig gebessert“ entlassen, arbeitete sie zu Hause, erlitt im September 1912 einen Unfall bei der Arbeit, der sie wieder in das Krankenhaus P. führte, wo sich alsbald Erscheinungen unzweifelhafter geistiger Erkrankung einstellten. (Der Unfall wurde als ,auslösendes Moment" betrachtet.) Es stellte sich eine schnell zunehmende, hochgradige Erregtheit ein, Verwirrtheit, Sinnestäuschungen, Personenverkennung, Neigung zu Unreinlichkeit und Zerstörungssucht. Am 15. X. 1912 nach Gábersee verbracht, war sie höchst erregt, motorisch-unruhig; sie lachte und schwätzte unaufhörlich verwirrtes Zeug, leierte Gebetsbruchstücke herunter, lachte und weinte in jähem Wechsel; das allgemeine Verhalten entsprach „durch ausgesprochen läppischen, zerfahrenen Charakter und gleichzeitige gemütliche Verödung dem Symptomenkomplex der Dementia praecox; Katalepsie und Befehlsautomatie zeitweise ziemlich ausgesprochen". Die Verblödung nahm rasch zu (März 1914): „.... ausgesprochene Affektleere; Pat. äußert nie Verlangen nach Entlassung, verhält sich Besuchen gegenüber völlig gleichgültig, hat kein Interesse an ihrer Umgebung, spricht spontan wenig. Vernachlässigt sich selbst gründlich; schmiert nachts im Isolierzimmer (wohin sie wegen nächtlicher Unruhe häufig gebracht werden muß) mit Kot, masturbiert viel, zerreißt Kleider und Bettstücke“. „Sie hat nur Interesse für Eßwaren, welche sie mit heißhungriger Gier verschlingt.“ Von Juli 1915 an bleibt das Krankheitsbild, das sich mit Sicherheit als eine Katatonie bezeichnen läßt, ziemlich stabil: immer besteht ausgesprochener Negativismus. „Pat. wechselt zwischen Zuständen von Erregtheit mit blindwütender, explosiv auftretender Gewalttätigkeit, verwirrtem Schreien und Schimpfen, Unreinlichkeit und Zerstörungssucht; und Zuständen von Apathie und stumpfem Hinbrüten, wobei sie blöde lächelnd, beinahe wie träumend, umhersteht." „Sie ist nie zu einer Beschäftigung zu bringen. Immer ist sie in Heißhunger bestrebt, alles EBbare und selbst unverdauliche Gegenstände (Baumzweigehen, Haare usw.) vom Boden aufzuheben und zu verschlingen." Von Mitte Mai 1916 an trat eine rasch zunehmende Tuberkulose der Lungen auf. Früher wurde Pat. als ,kräftig und gut aussehend" bezeichnet; von 17. V. 16 an wird das "fahle, eingefallene" Aussehen betont; zugleich damit werden die typischen Symptome der Lungentuberkulose angegeben: Husten, perkutor. Dämpfung über beiden Lungenspitzen, bronchiales Atmen usw. Das Fieber war äußerst unregelmäßig; zeitweise Typus inversus: morgendliche Steigerung bis $42,0^{\circ}$ - abendl. 37,3; Expektoration eitrigen, geballten Sputums. Dazu häuften sich Diarrhöen und zeitweises Erbrechen; letzteres hörte mit Darreichung von flüssiger Nahrung fast ganz auf. Abmagerung und Schwäche wurden schon Mitte Juni 1916 hochgradig; trotzdem - trotz Phthise und tuberkulöser Darmerkrankung - blieb der Heißhunger bestehen. Psychisch blieb Pat. unverändert; die Tuberkulose nahm den gewöhnlichen Verlauf bis zum Tode der Pat., der am 6. VIII. 1916 eintrat.

Die Sektion ergab vorgeschrittene Tuberkulose beider Lungen; beide Organe von pleuritischen Auflagerungen bedeckt, auf dem Durchschnitt zeigte sich nur mehr im linken Unterlappen normales, lufthaltiges Gewebe; alle übrigen Teile waren von erbsen- bis hühnereigroßen, verkästen Herden durchsetzt, in welche zahlreiche Kavernen bis zu Hühnereigröße eingestreut waren. Das Herz $(330,0 \mathrm{~g})$ hatte eine mürbe, schlaffe, fettig-degenerierte Muskulatur. Die Milz $(125,0 \mathrm{~g})$ 
sowie die Leber $(1320,0 \mathrm{~g})$ wiesen einzelne, im Parenchym zerstreute Tuberkuloseknötchen auf; die Leber war gänzlich verfettet. Die Nieren $(140,0 \mathrm{~g}$ und $145,0 \mathrm{~g})$ waren ohne wesentliche Veränderung. Der Dünndarm war nirgends verwachsen; seine Schleimhaut injiziert, im Coecum fand sich ein erbsengroßes, tuberkulöses Geschwür. Das Gehirn (1080,0 g) ließ außer beträchtlicher Flüssigkeitsansammlung im Subduralraum, verdickten und getrübten weichen Häuten makroskopisch nichts Besonderes nachweisen. Alle Organe waren äuBerst blutarm; das Fett an den betreffenden Organteilen geschwunden.

Der Magen lag (bei Eröffnung der Bauchhöhle) in situ zurückgesunken, nicht verwachsen mit der Umgebung und fühlte sich als derbe, harte Masse an. Bei der Eröffnung des Magens fand sich eine Fremdkörperansammlung, meist aus Holzwollfasern und Haaren bestehend, als eine harte, nicht zerfallende Masse, welche die Form des Magens angenommen hatte; seine Oberfläche war mit einem schleimigen Überzug versehen. Die Magenwand war dünn, am Fundus und der großen Kurvatur sichtlich verdünnt, die Schleimhaut nicht gefaltet, anscheinend atrophisch, nur am Pylorus war die Magenwand - abgesehen von dem normalerweise vorhandenen Ring - verdickt. Nahe der Kardia fand sich ein einmarkstückgroßes, schwarzbraunes Geschwür (Defekt der Magenschleimhaut), in der Nähe davon noch zwei kleinere, erbsengroße Geschwüre; die Magenwand um dieselben herum verdickt (bis $1 \mathrm{~cm}$ Durchschnitt). Außer der Fremdkörperansammlung fand sich im Magen nur etwas Schleim. Die Magenwand lag nicht gespannt um die Masse herum, welche übrigens ganz die Form des Magens (siehe Zeichnung) angenommen hatte. Das Pankreas erschien auffallend groß; doch ließen sich makroskopisch. keine krankhaften Veränderungen daran wahrnehmen (Abb. 3).

Die Fremdkörpermasse schien in diesem (III.) Falle den Magen nicht völlig - d.h. nicht für Flüssigkeiten - ausgefüllt und verstopft zu haben ;

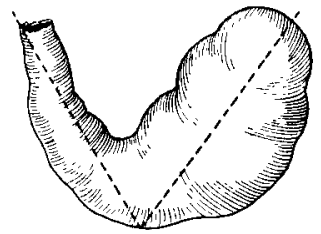

Abb. 3. es fehlte auch hier das im II. Falle so charakteristische Erbrechen; nur zeitweise trat solches auf neben den - weit häufigeren - Diarrhöen. Die Kranke erhielt ja auch seit mehreren Monaten nur mehr flüssige Kost; feste Nahrungsbestandteile würden sicher den Verschluß des Pylorus herbeigeführt haben, und hatten dies auch getan vor Darreichung der ausschließlich flüssigen Kost. Gerade hier hatten sich Geschwüre der Magenwand entwickelt im Gegensatz zu den Fällen I und II, bei welchen doch die Spannung der Magenwand über den Fremdkörpern eine deutlich nachweisbare war; freilich mochte bei Fall III das reichlich verschluckte eiterige Sputum die Entstehung von Geschwüren durch Infektion der Schleimhaut begünstigt haben.

Die Fremdkörperansammlung wurde im III. Falle zu Lebzeiten der Patientin nicht nachgewiesen, wegen der Unruhe und des Widerstrebens der Kranken, ferner, weil das charakteristische Erbrechen fehlte; vor allem aber deshalb, weil die Symptome der floriden Phthise die Aufmerksamkeit von sonstigen, vielleicht bemerkbaren Erscheinungen ablenkten. 
Bei der nochmaligen Betrachtung der drei Fälle muß sich vor allem die Frage aufdrängen, wie der Magen sich einer solchen Masse der verschiedenartigsten Fremdkörper gegenüber so reaktionslos, so untätig verhalten konnte, daß er geradezu ausgestopft wurde, ohne daß bei den Kranken schwere, subjektiv und objektiv auffallende Erscheinungen hervorgerufen wurden. (Selbstverständlich war in keinem der drei Fälle ein mechanisches Hindernis vorhanden - keine Verwachsung, Knikkung der benachbarten Darmabschnitte oder dgl., was einen Verschluß des Magens hätte herbeiführen können.) Aus den eingangs zitierten Fällen ist zu ersehen, wie schon einzelne Fremdkörper innerhalb relativ kurzer Zeit lebhafte Beschwerden hervorrufen können: Der Magen reagierte energisch auf die Anwesenheit der Fremdkörper, entweder durch heftige Schmerzen, welche die Operation notwendig machten, oder durch Entfernung der betreffenden Gegenstände per vias naturales, der einzige Fall, bei dem von einer Ansammlung von Fremdkörpern gesprochen werden konnte, betraf eine geisteskranke, an Melancholie leidende Frau. Und sicherlich ist Geisteskrankheit das wichtigste, ursächliche Moment. Von Hysterischen ist bekannt (Sch ma us s-Herxheimer), ,daß Haarballen ... im Magen von Hysterischen, ohne besondere Erscheinungen zu verursachen, sich finden". Aber in den vorliegenden drei Fällen handelt es sich nicht allein um das Ertragen von Fremdkc̈rpern, sondern es muß eine langdauernde Untätigkeit, eine förmliche Lähmung der Magenmuskulatur und der Innervation angenommen werden. Die Art der verschlungenen Fremdkörper kann dio Ursache sicher nicht sein - so, als würden Holzwolle, Haare, Leinwand und Wollstreifen leicht im Magen zurückgehalten; das Verschlingen von Fremdkörpern dieser Art konnte von mir bei mehreren anderen Geisteskranken beobachtet werden, bei denen später die Sektion keine Spur der nachweisbar verschluckten Dinge mehr vorfinden ließ: Diese waren per vias naturales ausgeschieden worden. Es sei hier noch in Kürze ein Fall mitgeteilt, bei welchem die genannte Art von Fremdkörpern den Magen passiert hatte, aber im Darm durch ein mechanisches Hindernis aufgehalten wurde.

Bach..., Th., 67 jähr. weibliche Kranke, wurde im Februar 1913 in G. aufgenommen mit der Diagnose Dementia senilis; sie war angeblich 3 Wochen vorher im Anschlusse an eine Hernienoperation akut erkrankt mit Verwirrtheit und hochgradiger Unruhe; es stellte sich heraus, daß sie schon mit 20 .Jahren geisteskrank (vorübergehend) gewesen sein soll. In der Anstalt war Pat. andauernd verwirrt, desorientiert, sehr laut und lärmend; abweisend und unzugänglich für jedermann, widerstrebend gegen alle Anforderungen, zerstörungssüchtig und unreinlich; aus ihren verwirrten Äußerungen war zu entnehmen, daß sie an Sinnestäuschungen litt (sie sah ,das Leiden Christi" vom Kruzifix an der Wand herunter auf sich zukommen), ferner daß schwere Störungen des Gedächtnisses, besonders der Merkfähigkeit nicht vorhanden waren. Ihr negativistisehes Widerstreben hielt bis zu ihrem Tode an. Der Gesamteindruck des psychischen Krankheitsbildes war 
weniger der einer Dementia senilis (Diagnose des Aufnahmezeugnisses) als der eines manisch-depressiven Irreseins. Die Kranke mußte ihrer Unruhe und Gewalttätigkeit wegen oft isoliert werden. Von September 1914 an magerte sie stark ab, litt an häufigen Diarrhöen, salivierte. Zu Anfang des Jahres 1915 mehrten sich die Diarrhöen; im April 1915 schien sich der Zustand zu bessern, bis Mitte Mai 1915 die Symptome einer inneren Darmeinklemmung, unstillbares Erbrechen, rapider Kräfteverfall und nach ca. 8 'Tagen der Tod der Pat. eintraten.

Die Sektion ergab an den Brust- und Bauchorganen außer den entsprechenden Alterserscheinungen nur in den Lungen einige wenige abgekapselte tuberkulöse Herde. Der Magen war klein, kontrahiert, leer. Der Darm war ,,im ganzen Bereiche des Dünndarms stark injiziert; der über $1 \mathrm{~m}$ lange, vom Coecum ca. $1 / 2 \mathrm{~m}$ entfernte Abschnitt bildete eine prallgefüllte, aufgetriebene, harte Masse, deren Peritoneumüberzug mißfarbig, von Auflagerungen bedeckt ist; sein Inhalt sind Fremdkörper (30-40 Stücke Wachsleinwand, Bettstück- und Rupfen- und Holzwollfasern usw.). Am distalen Ende der, wurstförmigen' Auftreibung ist der Darm stenosiert, geknickt, undurchgängig; die Darmwand dieser Stelle ist schwartenartig verdickt (bis $5 \mathrm{~mm}$ dick), die Schleimhaut weist zahlreiche, pfennigbis bohnengroße geschwürige Defekte auf". Die Knochen zeigten die Symptome der Osteomalacie: „das Promontorium typisch vorgewölbt und etwas nach rechts verbogen; alle Knochen, auch das Schädeldach, sind mit dem Messer leicht schneidbar; das Knochenmark ist braunrot, vorquellend, milzähnlich."

Diese Kranke Bach ..., Th., außerdem noch merkwürdig durch die Mitbeteiligung des Skelettes an der Ernährungsstörung (wie im Fall II), ist also eine der vielen Geisteskranken, welche Fremdkörper verschlingen, ohne daß im Magen eine Spur davon zurückbleibt und ohne daß die Menge der Fremdkörper geschätzt werden kann - wenn nicht, wie hier in der Darmstenose, ein besonderes Ereignis hinzukommt.

Krankhafter Heißhunger ist keine seltene Erscheinung (Kraepelin): ,Wir sehen bei paralytischen und katatonischen Kranken häufig eine sinnlose Gefräßigkeit sich einstellen, obwohl bei den wohlgenährten und trägen Kranken von einem wirklichen Nahrungsbedürfnis anscheinend keine Rede sein kann." Über das Triebhafte in der Natur dieses Heißhungers, des massenhaften Verschlingens von Fremdkörpern, besteht wohl kein Zweifel; dennoch erscheint es bemerkenswert und zugleich charakteristisch für die Natur des Heißhungers, daß in unseren drei Fällen die Anfüllung des Magens der Gefräßigkeit keinen Abbruch tut, daß der Heißhunger ungemindert andauerte zu einer Zeit, als der Magen bereits völlig ausgestopft und für feste - im Falle I und II auch für flüssige - Speisen ganz oder fast ganz undurchgängig war.

Die Anschoppung des Magens war bei den drei Fällen nur eine Teilerscheinung, nur ein einzelnes Symptom der tiefgehenden Ernährungsstörung: Die Abmagerung der Kranken, ihr schlechtes, erdig-fahles Aussehen begann schon früh, zu einer Zeit, als der Magen jedenfalls noch durchgängig war. Der Heißhunger der drei Kranken dauerte jahrelang an; die Patientinnen nahmen ihre eigenen Speisen und solche von Mitpatientinnen, soweit sie sie bekommen konnten - und das war damals 
vor dem Kriege und in der ersten Zeit des Krieges, sehr reichlich. Trotzdem besserte sich ihr Ernährungszustand nicht nur nicht, sondern es wurde eine beständige Abnahme der Ernährung beobachtet. Diese Erscheinung, der krankhafte Heißhunger, sowie die ständige Gewichtsabnahme ist bekannt und beschrieben bei hysterischen, paralytischen und katatonischen Kranken (Kr a e pelin III, S. 806, 817). Ein extremer Fall dieser Art ist angeführt bei Dr. Dre yfus: „Die Inanition im Verlauf von Geisteskrankheit": Ein Mann, der zu seinen täglichen Mahlzeiten das Fleisch von 15 Tauben und 3-4 Enten verschlang und selbe gleich nach der Mahlzeit wieder erbrach (ähnlich wie bei unserem Fall II); dieser Mann starb an Inanition; leider wurde die Sektion verweigert. Die Diagnose dieses zweifellos geisteskranken Mannes wurde auf Katatonie gestellt. An diesen, sowie den drei beschriebenen Fällen zeigt sich, daß die Magenfunktionen vom Zentralnervensystem aus gestört sind. Nicht nur bei Hysterischen, sondern gerade bei hebephrenen und katatonischen Erkrankungen finden sich Störungen, welche mit Ernährung und Stoffwechsel zusammenhängen, Störungen des Blutbildes, der Speichela bsonderung, solche vasomotorischer Art ( $\mathrm{K} \mathrm{r}$ a e peli in III, S. 750); auch Paresen der Schlingmuskulatur, Blasen- und Darmlähmungen werden angegeben (Knapp: ,Körperliche Symptome bei funktionellen Psychosen"). -

Bei unseren drei Fällen ist also eine vom Zentralnervensystem ausgehende Störung der reflektorischen Tätigkeit des Magens, geradezu eine Lähmung des Magens anzunehmen, neben schwerer Schädigung des Stoffwechsels und der gesamten Ernährung. Für letzteres spricht auch noch die Mitbeteiligung des Skelettes im Falle II: Die Erkrankung an Osteomalacie. Auch bei der nebenher erwäbnten Kranken Bach ..., Th., wurde eine vorgeschrittene Osteomalacie gefunden; ob es sich nicht auch hier um eine versteckte Dementia praecox, spez. Katatonie gehandelt hat, läßt sich leider - bei dem gänzlichen Fehlen der Anamnese - nicht mehr nachweisen; jedenfalls sind auch hier die drei Hauptmomente rereinigt: Geisteskrankheit, bzw. Erkrankung des Zentralnervensystems, schwere Emährungsstörung, Osteomalacie. Hier ist auf das häufige Vorkommen von Osteomalacie in der Anstalt Gabersee hinzuweisen, und zwar solcher, bei der ,... Paralyse, nachweisbar luetische Symptome, carcinomatöse oder tuberkulöse Knochenerkrankungen ausgeschlossen sind" (Dr. Imhof: „Uber Geisteskrankheit und Osteomalacie); unter den 15 aufgezählten Fällen befanden sich 10 Dementia praecox (3 Dementia senilis und 2 Imbezille; 2 männliche und 13 weibliche). Utber die Verbindung von Dem. praecox und Osteomalacie sagt Krae pelin (I, S. 80): „In einzelnen Füllen scheint sich die Osteomalacie mit Dementia praecox zu verbinden ... ob es sich um mehr als zufälliges Zusammentreffen handle, ist zweifelhaft." 
Bemerkenswert ist vielleicht auch das Verhalten der Magenschleimhaut in den drei Fällen: Obwohl dieselbe durch die große und scbwere Fremdkörpermasse relativ lange Zeit gezerrt und gedrückt wurde, fand sich doch bei I und II keine Spur einer Gangrän, eines Defektes, der als Druckbrand hätte bezeichnet werden können; bei III waren wohl derartige Stellen vorhanden, aber hier war es wohl die Tuberkulose, oder vielmehr das massenhaft verschluckte eitrige Sputum, welches hauptsächlich die angegebene Geschwürbildung verursachte; bei der sonstigen schweren Ernährungsstörung eine immerhin auffallende Erscheinung. (Man denkt hierbei daran, wie selten im allgemeinen Decubitus bei stuporösen, unbeweglich daliegenden Dementia-praecox-Kranken auftritt, im Gegensatz zu den Paralytikern!) Ebenso auffallend ist die Tatsache, daß bei unseren Fällen trotz des enorm vermehrten Gewichtes (während längerer Zeit) eine eigentliche Enteroptose, bzw. Gastroptose nicht zu konstatieren war; der untere Rand des Magens reichte zwar bis unter den Nabel herab, aber nur infolge des vergrößerten Magenvolumens; ein Beweis - wenn es eines solchen bedürfte - daß die Ätiologie der Gastro-, bzw. Enteroptose auf andere als mechanische Ursachen zurückzuführen ist (Dr. Schwerdt: ,Ätiologie der Enteroptose $\left.{ }^{6}\right)$.

Noch eine Folgerung mehr praktischer Natur ließe sich dem im vorausgehenden Angegebenen entnehmen, nämlich, daß erregte und unreinliche Verblödete sogar durch so ungefährliche und zugleich unumgänglich notwendige Dinge, wie Holzwolle und Wachsleinwand, geschädigt werden können. Besonders die Holzwollfasern hatten sich - ganz wie Haarbüschel - ineinander verfilzt und so, zugleich mit den Wachsleinwandstreifen, Wollfäden usw., den stets wachsenden Tumor erzeugt. Die Holzwolle wird anscheinend von den Verdauungssäften wenig oder nicht angegriffen; außerdem steht sie den Kranken in größeren Mengen zu Gebote und kann, namentlich bei Nacht und im Isolierzimmer, leicht unbemerkt verzehrt werden.

\section{Literaturverzeichnis.}

Kraepelin, Psychiatrie. 1909.

Schmauss - Herxheimer, Pathol. Anatomie. 1912.

Archiv für Psychiatrie 41: Dr. Dre yfus, ,Inanition im Verlauf von Geisteskrankheiten“. 44: Dr. Knapp, „Körperliche Symptome bei funktionellen Psychosen".

Zeitschrift für gesamte Neurologie und Psychiatrie 14, 2: Dr. Imhof, ,Über Osteomalacie und Geisteskrankheit":

Deutsche medizinische Wochenschrift 1897: Dr. Fricken, „Ein älterer Fall von Fremdkörpern im Magen“; 1896: Dr. Schwerd t, "Ätiologie der Enteroptose“. Langenbecksches Archiv 33, 574. 